\title{
Comparison of Immunohistochemistry and Conventional Stains for Helicobacter Pylori Detection in Gastric Biopsies of Patients Receiving Proton Pump Inhibitors
}

\author{
Kongsak Loharamtaweethong, M.D., Napaporn Puripat, M.D.
}

Department of Anatomical Pathology, Faculty of Medicine, Vajira Hospital, Navamindradhiraj University, Dusit, Bangkok 10300, Thailand.

Received 6 January 2020 • Revised 1 May 2020 • Accepted 1 May 2020 • Published online 3 July 2020

\begin{abstract}
:
Objective: To evaluate the diagnostic performance of different staining methods for Helicobacter pylori (H. pylori) in patients treated with proton pump inhibitors (PPI)

Material and Methods: This is a retrospective study involving 75 gastric biopsies from patients with and without PPI therapy between October 2018 and September 2019. Slides were stained with hematoxylin and eosin (H\&E); Giemsa and immunohistochemical staining $(\mathrm{IHC})$ was done using polyclonal anti-H. pylori antibodies. Statistical analysis was performed to determine any association between the results of different staining methods and PPI consumption. Sensitivity, specificity and positive-negative predictive values of H\&E and Giemsa stains were calculated.
\end{abstract}

Results: Overall, $H$. pylori infection was detected in $33.3 \%$ of patients using $I H C, 26.7 \%$ using $H \& E$, and $28.0 \%$ via Giemsa staining. A strong correlation was found between PPI consumption and low $H$. pylori density detected via IHC ( $p$-value=0.015) but not using H\&E and Giemsa staining. The sensitivity of H\&E and Giemsa was markedly reduced as a result of PPI consumption ( $57.1 \%$ to $18.2 \%$ vs. $85.7 \%$ to $27.3 \%$, respectively).

Conclusion: Identification of $\mathrm{H}$. pylori using IHC in patients treated with PPI was superior to H\&E and Giemsa stains. Both H\&E and Giemsa stains showed a marked decrease in sensitivity in patients receiving PPI. The results indicate that IHC should be performed in patients with PPI therapy instead of H\&E or Giemsa stains, and PPI should be discontinued for at least 14 days before the performance of endoscopy.

Keywords: conventional stains, H. pylori, immunohistochemistry, proton pump inhibitors

Contact: Kongsak Loharamtaweethong, M.D. Department of Anatomical Pathology, Faculty of Medicine, Vajira Hospital, Navamindradhiraj University, Dusit, Bangkok 10300 Thailand.

E-mail: Kongsakloharamtaweethong@hotmail.com

This is an open access article under the CC BY-NC-ND license

(http://www.jhsmr.org/index.php/jhsmr/about/editorialPolicies\#openAccessPolicy).

J Health Sci Med Res 2020;38(4):321-330 doi: $10.31584 /$ jhsmr.2020752 www.jhsmr.org 


\section{Introduction}

Diseases related to Helicobacter pylori (H. pylori) affect more people globally than any other disorder; it is believed that over $50.0 \%$ of the world's population is infected to date. ${ }^{1}$ Warren and Marshall take credit for the discovery of $H$. pylori in 1983 when they examined the gastric mucosal biopsy specimens obtained from patients suffering from peptic ulcers or chronic active gastritis. ${ }^{2} H$. pylori is a Gram negative spiral organism ${ }^{3}$ believed to cause peptic ulcers, chronic gastritis, and gastric cancer. Moreover, it could be responsible for nonHodgkin B cell lymphoma, especially mucosa-associated lymphoid tissue lymphoma. Clinically, a number of different invasive techniques (requiring endoscopy and biopsy) or non-invasive techniques (including serology, respiratory urea breath test, or the detection of fecal antigen) can be performed in order to detect $H$. pylori infection.

In routine practice, hematoxylin and eosin (H\&E), Giemsa, and immunohistochemistry staining methods are commonly used in the identification of $H$. pylori after an endoscopic procedure. The H\&E stain can detect $H$. pylori under light microscopy; however, it becomes difficult to identify $H$. pylori when it is present in low density. Although the Giemsa stain is a more popular method than H\&E in many laboratories, it could lead to false negative results when the organisms are few.

Furthermore, $H$. pylori bacteria could exist in two forms-rod and coccoid shapes. The rod-shaped bacteria often change to coccoid-shaped ones when undergoing proton pump inhibitors (PPI) therapy. ${ }^{4,5}$ It is difficult to determine the presence of coccoid-shaped bacteria when the Giemsa staining technique is applied because it can be very hard to distinguish the coccoid-shaped bacteria from other bacterial forms or other matter. Consequently, the best approach to use if $H$. pylori is to be detected is immunohistochemistry staining. Moreover, it is believed that bacterial transmission could be facilitated by the coccoid- shaped $H$. pylori, and that these bacteria could play a key role when infection recurs following the completion of antimicrobial therapy as well. Moreover, PPI could also lead to false-negative results in other tests. ${ }^{6}$ Owing to their in vitro anti-H. pylori activity, ${ }^{7} \mathrm{PPI}$ could reduce the bacterial load ${ }^{8-10}$ and suppress the urease activity of H. pylori. ${ }^{11}$ Overall, the Immunohistochemical (IHC) stain has advantages over the other methods when the biopsied specimens have a few coccoid or intracellular forms of $H$. pylori present. Furthermore, it has a high specificity as it can differentiate between $\mathrm{H}$. pylori and other mimicking organisms. ${ }^{12}$

Hence, in this study, we aimed to evaluate the diagnostic value of the immunohistochemical method using polyclonal anti-H. pylori antibodies as a gold standard test for the identification of these organisms in $\mathrm{PPI}-$ treated and $\mathrm{PPI}-$ untreated patients and compare its results with those of routine histochemical methods like H\&E and Giemsa stains. The histopathological examination of gastric biopsies was reported in accordance with the "Updated Sydney System" classification of chronic gastritis. ${ }^{13}$

\section{Material and Methods}

In this retrospective 1 year study, the gastric biopsies taken from patients with a histopathological diagnosis of gastritis, who underwent an endoscopic examination between October 2018 and September 2019 at the Vajira Hospital, Faculty of Medicine, Navamindradhiraj University, were studied. Patients were divided into two groups according to PPI therapy; patients receiving PPI during the 14-day period prior to the procedure (called the PPI-treated group) and those receiving no PPI for at least 14 days before endoscopy (called the PPI-untreated group). At least four biopsy specimens were collected from both the antrum and corpus during each endoscopy. The clinical data were reviewed and recorded. The study protocol complied with the principles of the Declaration of Helsinki and was approved by the Institutional Ethics Committee of 
Navamindradhiraj University. Four-micron-thick sections were placed on slides for histochemistry. After that, the histological sections were dyed with H\&E and Giemsa following our routine protocols. Immunohistochemistry was performed on the Leica Bondmax platform (Leica Micro-systems, Buffalo Grove, Illinois, the United States of America) on 4- $\mu$ m-thick consecutive sections in accordance with the manufacturer's instructions using rabbit polyclonal anti- $H$. pylori antibodies (1:150, Novocastra). In the present study, IHC was considered as the gold standard for $H$. pylori detection. All samples were examined and diagnosed by two experienced histopathologists unaware of the patient's previous diagnosis. Specimens showing a positive result for IHC were deemed to be $H$. pylori positive. Gastritis was classified in accordance with the updated Sydney system.

Bacterial density was quantified by counting Helicobacter-like organisms on the mucosal surface under a high-power field. The four grades used for bacterial density were: normal (no bacteria); mild (individual bacteria or small groups on less than a third of the mucosal surface); moderate (bacteria present in numbers greater than 'mild' but fewer than 'severe'), and severe (large groups of bacteria on more than two thirds of the mucosal surface). These classifications were derived from the visual analogue scale of the updated Sydney system.

For statistical purposes, all cases were grouped according to the presence or absence of (1) chronic gastritis, (2) inflammatory activity, and (3) $H$. pylori and/or chronic gastritis- related morphological alterations of the gastric mucosa. The considered morphological changes included intestinal metaplasia, atrophy, ulcer and foveolar hyperplasia. Briefly, the different subgroups were as follows: (1) chronic active gastritis with morphological change, (2) chronic active gastritis without morphological change, (3) chronic nonactive gastritis with morphological change, and (4) chronic nonactive gastritis without morphological change.
The chi-square or Fisher's exact tests were used to evaluate differences between categorical variables. The sensitivity, specificity, positive predictive value (PPV), and negative predictive value (NPV) of the tests were also calculated. The statistical analysis was carried out using the Stata software (version 13, StataCorp LP, College Station, TX).

In the present study, 75 gastric biopsies obtained from October 2018 to September 2019 and histopathologically diagnosed as chronic gastritis via the H\&E stain were included for investigation. The demographic and histopathological characteristics of the study population are illustrated in Table 1. Of the 75 studied patients, 35 (46.7\%) were male and 40 (53.3\%) female, the mean age was $64.7 \pm 3.0$ years, and 33 (44.0\%) underwent PPI therapy and 42 (56.0\%) did not.

The mean age was $64.0 \pm 4.5$ years for the PPItreated patients, and $65.2 \pm 4.0$ years for the PPIuntreated patients. No statistically significant correlation was observed between PPI-treated and untreated groups with regard to patient age, sex, and histopathological characteristics as shown in Table 1.

\section{Comparison of the diagnostic performances}

\section{of the different staining methods}

Among the 75 patients studied, the IHC stain could identify bacilli in 25 (33.3\%) biopsies, and the Giemsa and H\&E stains, in 21 (28.0\%) and 20 (26.7\%) biopsies, respectively (Table 2 ). In 10 cases, $H$. pylori was suspected by the H\&E stain, but the results were negative in the IHC stain. Overall, the results of the H\&E and Giemsa stains were consistent with those of the $\mathrm{IHC}$ stain in 50 $(66.7 \%)$ and $59(78.7 \%)$ specimens, respectively. There were 6 cases that showed no bacilli in the $\mathrm{IHC}$ stain although they were suspected to be positive by the Giemsa stain. The overall sensitivity and specificity of the H\&E stain were $40.0 \%$ and $80.0 \%$, respectively. The 
Table 1 Classification of 75 patients according to age, sex, histopathological findings, and the results of Helicobacter pylori diagnosis according to hematoxylin and eosin stain, Giemsa and immunohistochemical staining

\begin{tabular}{|c|c|c|c|}
\hline Characteristics & $\begin{array}{l}\text { PPI-treated } \\
(n=33)\end{array}$ & $\begin{array}{l}\text { PPI-untreated } \\
(n=42)\end{array}$ & $p$-value \\
\hline Mean age (years) & $64.0 \pm 4.5$ & $65.2 \pm 4.0$ & \\
\hline \multicolumn{4}{|l|}{ Gender } \\
\hline Male & $14(42.4)$ & $21(50.0)$ & 0.514 \\
\hline Female & $19(57.6)$ & $21(50.0)$ & \\
\hline \multicolumn{4}{|l|}{ Histology } \\
\hline Chronic active gastritis with morphological change & $2(6.1)$ & $5(11.9)$ & 0.385 \\
\hline Chronic active gastritis without morphological change & $11(33.3)$ & $9(21.4)$ & \\
\hline Chronic nonactive gastritis with morphological change & $2(6.1)$ & $2(4.8)$ & \\
\hline Chronic nonactive gastritis without morphological change & $18(54.6)$ & $26(61.9)$ & \\
\hline \multicolumn{4}{|l|}{ H. pylori diagnosis } \\
\hline \multicolumn{4}{|l|}{ H\&E } \\
\hline Positive & $6(18.2)$ & 14 (33.3) & 0.141 \\
\hline Negative & $27(81.8)$ & $28(66.7)$ & \\
\hline \multicolumn{4}{|l|}{ Giemsa } \\
\hline Positive & $4(12.1)$ & $17(40.5)$ & $0.009^{*}$ \\
\hline Negative & $29(87.9)$ & $25(59.5)$ & \\
\hline \multicolumn{4}{|l|}{$\mathrm{IHC}$} \\
\hline Positive & 11 (33.3) & 14 (33.3) & 1.000 \\
\hline Negative & $22(66.7)$ & $28(66.7)$ & \\
\hline
\end{tabular}

Values are presented as number (\%) or mean \pm standard deviation.

$\mathrm{H} \& \mathrm{E}=$ hematoxylin and eosin stain, $\mathrm{IHC}=$ immunohistochemical stain, $\mathrm{PPI}=$ proton pump inhibitors, $\mathrm{H}$. pylori=Helicobacter pylori ${ }^{*} \mathrm{p}$-value $<0.050$ (statistically significant)

Table 2 Overall detection rate of Helicobacter pylori with hematoxylin and eosin stain, Giemsa and immunohistochemical staining

\begin{tabular}{ll}
\hline Methods & Number of patients (\%) \\
\hline H\&E & \\
Negative & $55(73.3)$ \\
Positive & $20(26.7)$ \\
Total & $75(100.0)$ \\
Giemsa & \\
Negative & $54(72.0)$ \\
Positive & $21(28.0)$ \\
Total & $75(100.0)$ \\
IHC & \\
Negative & $50(66.7)$ \\
Positive & $25(33.3)$ \\
Total & $75(100.0)$ \\
\hline
\end{tabular}

$\mathrm{H} \& \mathrm{E}=$ hematoxylin and eosin stain, $\mathrm{IHC}=$ immunohistochemical stain sensitivity (60.0\%) and specificity (88.0\%) of the Giemsa stain were higher than those of the H\&E stain. However, in the subgroup analysis, using immunohistochemistry as the gold standard, we found that a significantly higher percentage of patients with PPI use had a mild $H$. pylori density when compared to patients without PPI use (81.8\% vs. $28.6 \%$, respectively; $p$-value $=0.015)$. In contrast, no significant difference in $H$. pylori density was observed between the H\&E and Giemsa stains ( $p-$ value=1.000 vs. $p$-value $=1.000$, respectively) (Table 3 ).

Considering $\mathrm{IHC}$ stain as the gold standard for the identification of $H$. pylori in gastric mucosal biopsies in the PPI-untreated group, the sensitivity and specificity of the H\&E stain were $57.1 \%$ and $78.6 \%$, respectively. The sensitivity $(85.7 \%)$ and specificity $(82.1 \%)$ of the Giemsa 
stain were higher than those of the H\&E stain. Similarly, the positive and negative predictive values of the $\mathrm{H} \& \mathrm{E}$ stain were lower than those of Giemsa stain $(57.1 \%$ vs. $70.6 \%$ and $78.6 \%$ vs. $92.0 \%$, respectively). In contrast, the sensitivity of the H\&E and Giemsa stains dramatically dropped to $18.2 \%$ and $27.3 \%$, respectively, in the PPItreated group, whereas their specificity increased slightly to $81.8 \%$ and $95.5 \%$, respectively. The comparative results of the H\&E, Giemsa, and IHC stains are show in Figures 1A-C and summarized in Tables 4 and 5).

Table 3 Helicobacter pylori density comparison between hematoxylin and eosin stain and Giemsa with immunohistochemical as the gold-standard test

\begin{tabular}{llll}
\hline Method/H. pylori density & PPI-treated & PPI-untreated & p-value \\
\hline H\&E & $3(50.0)$ & $7(50.0)$ & 1.000 \\
Mild & $3(50.0)$ & $7(50.0)$ & \\
$\quad$ Moderate/marked & $1(25.0)$ & $7(41.2)$ & 1.000 \\
Giemsa & $3(75.0)$ & $10(58.8)$ & $0.015^{*}$ \\
Mild & & & \\
Moderate/marked & $9(81.8)$ & $1(28.6)$ & $10(71.4)$ \\
IHC & $2(18.2)$ & & \\
Mild & & \\
Moderate/marked &
\end{tabular}

Values are presented as number (\%) or mean \pm standard deviation.

$\mathrm{H} \& \mathrm{E}=$ hematoxylin and eosin stain, $\mathrm{IHC}=$ =immunohistochemical stain, $\mathrm{PPI}=$ proton pump inhibitors, $\mathrm{H}$. pylori=Helicobacter pylori ${ }^{*} p$-value $<0.050$ (statistically significant)

Table 4 Comparison of hematoxylin and eosin stain, Giemsa and immunohistochemical staining methods

\begin{tabular}{|c|c|c|c|c|}
\hline \multirow{2}{*}{ Methods and results } & \multicolumn{2}{|c|}{ H\&E } & \multicolumn{2}{|c|}{ Giemsa } \\
\hline & Positive & Negative & Positive & Negative \\
\hline \multicolumn{5}{|l|}{ All patients } \\
\hline \multicolumn{5}{|l|}{$\mathrm{IHC}$} \\
\hline Positive & 10 & 15 & 15 & 10 \\
\hline Negative & 10 & 40 & 6 & 44 \\
\hline \multicolumn{5}{|l|}{ PPI-treated group } \\
\hline \multicolumn{5}{|l|}{$\mathrm{IHC}$} \\
\hline Positive & 2 & 9 & 3 & 8 \\
\hline Negative & 4 & 18 & 1 & 21 \\
\hline \multicolumn{5}{|l|}{ PPI-untreated group } \\
\hline \multicolumn{5}{|l|}{$\mathrm{IHC}$} \\
\hline Positive & 8 & 6 & 12 & 2 \\
\hline Negative & 6 & 22 & 5 & 23 \\
\hline
\end{tabular}

$\mathrm{H} \& \mathrm{E}=$ hematoxylin and eosin stain, $\mathrm{IHC}=$ immunohistochemical stain, $\mathrm{PPI}=$ proton pump inhibitors 

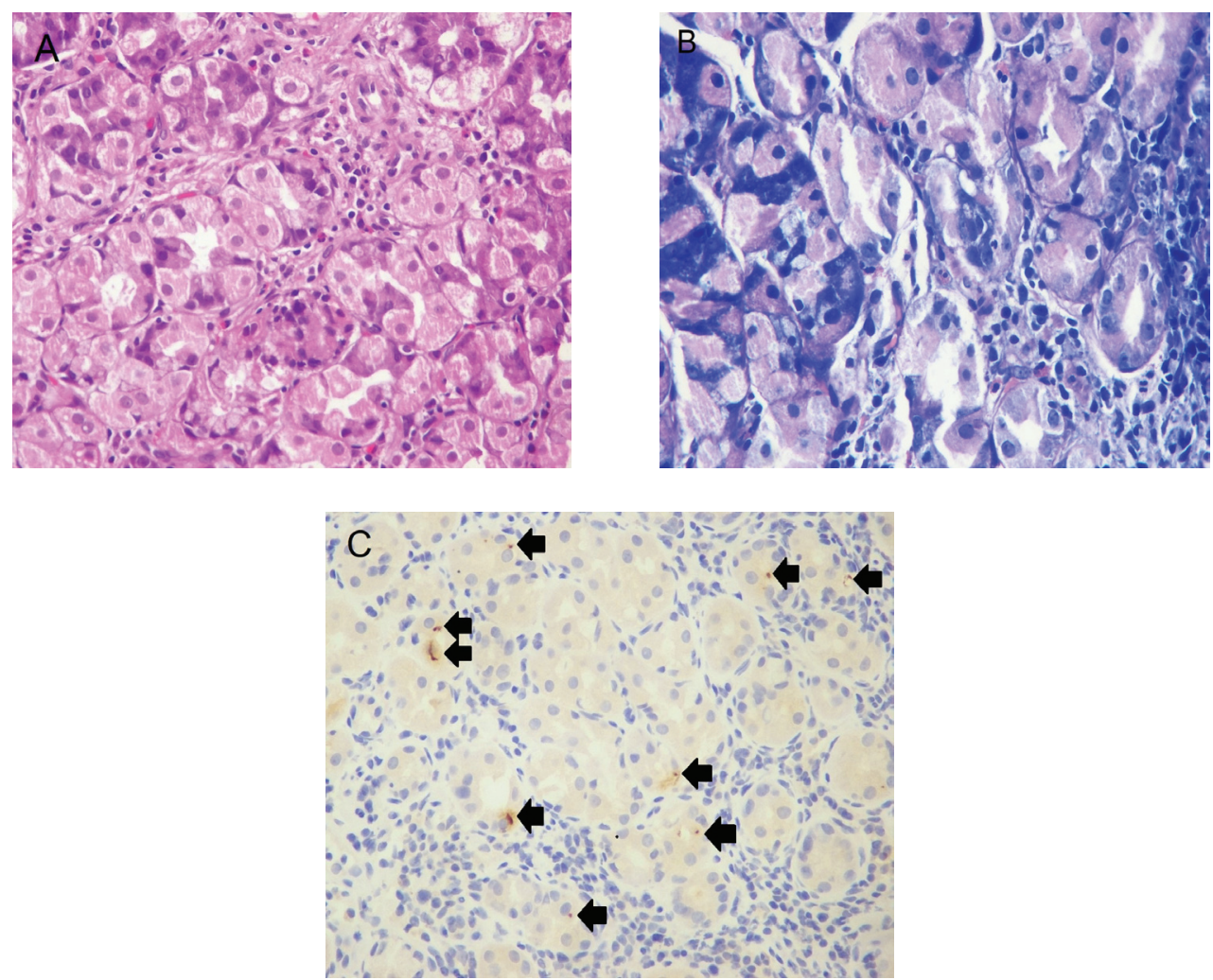

Figure $1 \mathrm{~A}$ case of PPI-treated patient showing no organisms on H\&E (A) on Giemsa stain (B). IHC staining (C) showing focal positivity with few $H$. pylori (arrows) (original magnification $\times 40$ ).

Table 5 Sensitivity, specificity, positive-negative predictive values of hematoxylin and eosin stain and Giemsa

\begin{tabular}{lllll} 
All patients & & & \\
\hline Method & Sensitivity (\%) & Specificity (\%) & Positive predictive value (\%) & Negative predictive value (\%) \\
\hline H\&E & 40.0 & 80.0 & 50.0 & 72.7 \\
Giemsa & 60.0 & 88.0 & 71.4 & 81.5
\end{tabular}

PPI-treated group

\begin{tabular}{lllll}
\hline Method & Sensitivity $(\%)$ & Specificity $(\%)$ & Positive predictive value $(\%)$ & Negative predictive value $(\%)$ \\
\hline H\&E & 18.2 & 81.8 & 33.3 & 66.7 \\
Giemsa & 27.3 & 95.5 & 75.0 & 72.4 \\
\hline
\end{tabular}


Table 5 (continued)

\begin{tabular}{lllll} 
PPI-untreated group & & & \\
\hline Method & Sensitivity $(\%)$ & Specificity $(\%)$ & Positive predictive value $(\%)$ & Negative predictive value (\%) \\
\hline H\&E & 57.1 & 78.6 & 57.1 & 78.6 \\
Giemsa & 85.7 & 82.1 & 70.6 & 92.0 \\
\hline
\end{tabular}

$\mathrm{H} \& \mathrm{E}=$ hematoxylin and eosin stain, $\mathrm{PPI}=$ proton pump inhibitors

\section{Discussion}

In the present study, the $H$. pylori infection rate was $33.3 \%$ by immunohistochemistry, $28.0 \%$ by Giemsa, and $26.7 \%$ by H\&E. Among the 75 patients, spiral bacterial cells were visualized in $20(26.7 \%)$ cases using $\mathrm{H} \& \mathrm{E}$, in 21 using Giemsa (28.0\%), and in $25(33.3 \%)$ using IHC. The H. pylori infection rate in the present study was relatively lower than those reported by previous studies. The study conducted by Pérez-Pérez et al. ${ }^{14}$ showed that the prevalence of $H$. pylori infection increased with age-from $17.5 \%$ among children aged 5 to 9 years old to $55.0 \%$ among those in their third decade of life; the infection peak was at $75.0 \%$ among those 30 to 49 years of age. Another study from Thailand conducted by Chinprasatsak et al. ${ }^{15}$ discovered a relatively high $H$. pylori infection rate, up to $73.8 \%$, compared to our study. However, many recent studies have shown a decreasing trend for the $H$. pylori infection rate in some areas of the world. It has been hypothesized that this reduction might result from a better awareness of public health, improvements in personal hygiene, effective diagnostic tests as well as the eradication of $H$. pylori, which make it difficult to analyze the data between different populations. ${ }^{16,17}$

Regarding $H$. pylori detection methods, the H\&E, Giemsa and immunohistochemistry staining techniques have been commonly used for the identification of organisms in gastric mucosal biopsies. Though several methods for the identification of these bacilli have been investigated, the identification of $H$. pylori by histological methods in gastric biopsies remains the most common and the most effective test. ${ }^{18}$ The H\&E stain could detect $H$. pylori under high magnification, but it becomes difficult to identify $H$. pylori when it is present at a low density and when atrophic mucosal changes are present. The Giemsa stain is the preferred method over H\&E in many laboratories; however, it could lead to false negative results when the organisms are few or in patients with a prior incomplete treatment for $H$. pylori gastritis. IHC stains enjoy some advantages when patients experience a partial treatment for $\mathrm{H}$. pylori gastritis or either have few or coccoid forms of H. pylori present. This method has a high specificity because it can differentiate between other mimicking organisms and can, therefore, be considered the gold standard test. ${ }^{19}$ It is noteworthy that $\mathrm{IHC}$ has been found to show a good performance with a sensitivity of $89.6 \%$, specificity of $100.0 \%$, PPV of $100.0 \%$ and NPV of $84.1 \% .^{20}$ Furthermore, the proportions of PPI patients with negative results via H\&E and Giemsa satins ( $81.8 \%$ and $87.9 \%$, respectively) were considerably higher than those of non-PPI patients $(66.7 \%$ and $59.5 \%$, respectively). Meanwhile, the IHC technique yielded the same proportions in both patients who received PPI $(66.7 \%)$ and those who did not $(66.7 \%)$. Considering $H$. pylori density, there was a discrepancy in $H$. pylori density when using $\mathrm{IHC}$ as the gold standard test. PPI-treated patients showed a higher prevalence of mild $H$. pylori density when compared to patients without 
PPI treatment (81.8\% vs. 28.6\%; p-value<0.050). No such correlation was observed in the H\&E or Giemsa staining methods.

In this study, on the basis of the results obtained from the H\&E and Giemsa stainns, the Giemsa staining had a higher sensitivity and specificity than H\&E in patients who did not take PPI (sensitivity: $85.7 \%$ vs. $57.1 \%$; specificity: $82.1 \%$ vs. $78.6 \%$ ). However, we found that the sensitivity of the H\&E and Giemsa stains dramatically dropped in patients with PPI use. The alterations in sensitivity were more prominent in both $\mathrm{H} \& \mathrm{E}$ and Giemsa (from $57.1 \%$ and $85.7 \%$ to $18.2 \%$ and $27.3 \%$, respectively) due to $\mathrm{PPI}$ use. Our results indicated that in patients who did not receive PPI, Giemsa had the best performance of the two methods-sensitivity $85.7 \%$, specificity $82.1 \%$, PPV $70.6 \%$, and NPV $92.0 \%$.

In general, the Giemsa stain was superior to H\&E in the detection of bacilli. The major disadvantage of this stain is that it produces little contrast between bacteria and the surrounding tissue. However, the presence of a few bacteria and the identification of coccoid forms of bacilli make identification difficult via both the routine and the histochemical methods. Nevertheless, these difficulties are not encountered when identifying bacilli using the IHC method. In the present study, among the stains utilized, the IHC stain identified bacilli in higher proportion of the PPI-positive biopsies. The IHC stain yielded a positive result in $33.3 \%$ of $\mathrm{PPI}$-positive biopsies, whereas the H\&E and Giemsa stains identified organisms in $18.2 \%$ and $12.1 \%$ of cases, respectively.

PPI are known to serve as bactericidal agents for $H$. pylori. ${ }^{21}$ The $\mathrm{pH}$ level in the stomach is increased by PPIs, whereupon the $H$. pylori urease produces ammonia, which is then accumulated and, thus, effectively curtailing the viability of the bacteria. ${ }^{22}$ As a consequence of exposure to PPI and antibiotics, the spiral forms of $H$. pylori will turn in a coccoid shape and exhibit a reduced urease activity. ${ }^{23}$ In vitro experiments have demonstrated that the inhibitory influence of PPI on $H$. pylori growth is dependent upon the concentrations involved, ${ }^{21}$ while the bacterium's virulence properties could have an effect as well. ${ }^{24}$ At concentrations of $\leq 0.625 \mu \mathrm{g} / \mathrm{ml}$, lansoprazole has been shown to exhibit bactericidal properties and be able to inhibit growth. Accordingly, it can be concluded that the effects of PPI on the $H$. pylori rapid urea test and culture are dependent upon the type of PPI and the dosage taken by patients. ${ }^{25}$

In the present study, we found that the IHC staining method for $H$. pylori detection was significantly affected by PPI consumption. The IHC method revealed a low bacterial density in the majority of patients with PPI use. Consequently, $H$. pylori might not be detected by the H\&E and Giemsa stains because of the rare bacteria. The IHC stain was considered the gold standard for the identification of bacilli in our study, while the H\&E and Giemsa stains showed a sensitivity of $18.2 \%$ and $27.3 \%$ and a specificity of $81.8 \%$ and $95.5 \%$, respectively. In some countries, PPI are frequently prescribed, even in scenarios where it might not be necessary such as mild dyspepsia. ${ }^{26}$ Thailand is one such country, with patients taking PPI for subclinical or mild gastritis. As a result, diagnostic testing (H\&E, Giemsa stains) for H. pylori often generates false negative outcomes. It is, therefore, suggested that an endoscopy should be carried out no earlier than two weeks after the cessation of PPI consumption $^{27}$, and IHC should be performed in all cases instead of H\&E or Giemsa in order to avoid false negative results, especially in patients with PPI use.

\section{Conclusion}

H\&E and Giemsa stains showed a marked decrease in sensitivity to detect $H$. pylori in patients receiving PPI. The ability of $\mathrm{IHC}$ to identify $H$. pylori was superior to those of both H\&E and Giemsa stains. Hence, 
IHC should be preferred in patients treated with PPI instead of H\&E and Giemsa stains.

\section{Acknowledgement}

The authors would like to thank the following colleagues at the Department of Anatomical Pathology, Navamindradhiraj University: Ms. Sujitra Tanvanich, Mrs. Unaporn Sitthivilai, and Mrs. Pornpimon Kongjan for their support and technical help regarding the study.

\section{Funding sources}

This study was supported by a grant from the Navamindradhiraj University Research Fund.

\section{Conflict of interest}

The authors declare that they have no conflict of interest.

\section{References}

1. Go MF. Review article: natural history and epidemiology of Helicobacter pylori infection. Aliment Pharmacol Ther 2002;16: 3-15.

2. Marshall B, Adams PC. Helicobacter pylori: a nobel pursuit? Can J Gastroenterol 2008;22:895-89.

3. Garg B, Sandhu V, Sood N, Sood A, Malhotra V. Histopathological analysis of chronic gastritis and correlation of pathological features with each other and with endoscopic findings. Pol J Pathol 2012;3:172-8.

4. Mizoguchi H, Fujioka T, Kishi K, Nishizono A, Kodama R, Nasu M. Diversity in protein synthesis and viability of Helicobacter pylori coccoid forms in response to various stimuli. Infect Immun 1988;66:5555-60.

5. Dhakhwa R, Acharya IL, Shresta HG. Histopathologic study of chronic antral gastritis. J Nepal Health Res Counc 2012;10: 57-60.

6. Malfertheiner P, Megraud F, O'Morain CA, Gisbert JP, Kuipers EJ, Axon AT, et al. Management of Helicobacter pylori infection-The Maastricht V/Florence Consensus Report. Gut 2017;66:6-30.

7. Saniee P, Shahreza S, Siavoshi F. Negative effect of proton- pump inhibitors (PPIs) on Helicobacter pylori growth, morphology, and urease test and recovery after PPI removal-an in vitro study. Helicobacter 2016;21:143-52.

8. Gatta L, Vakil N, Ricci C, Osborn JF, Tampieri A, Perna F, et al. Effect of proton pump inhibitors and antacid therapy on 13C urea breath tests and stool test for Helicobacter pylori infection. Am J Gastroenterol 2004;99:823-9.

9. Graham DY, Genta R, Evans DG, Reddy R, Clarridge JE, Olson CA, et al. Helicobacter pylori does not migrate from the antrum to the corpus in response to omeprazole. Am J Gastroenterol 1996;91:2120-4.

10. Suzuki M, Suzuki H, Kitahora T, Miyazawa M, Nagahashi S, Suzuki K, et al. Treatment with a proton pump inhibitor promotes corpus gastritis in patients with Helicobacter pyloriinfected antrum-predominant gastritis. Aliment Pharmacol Ther 2002;16:159-65.

11. Graham DY, Opekun AR, Hammoud F, Yamaoka Y, Reddy R, Osato MS, et al. Studies regarding the mechanism of false negative urea breath tests with proton pump inhibitors. Am J Gastroenterol 2003;98:1005-9.

12. Yup LJ, Nayoung K. Diagnosis of Helicobacter pylori by invasive test: histology. Ann Transl Med 2015;3:1-8.

13. Dixon MF, Genta RM, Yardley JH, Correa P. Classification and grading of gastritis. The updated Sydney System. International Workshop on the Histopathology of Gastritis, Houston 1994. Am J Surg Pathol 1996;20:1161-81.

14. Pérez-Pérez Gl, Taylor DN, Bodhidatta L, Wongsrichanalai J, Baze WB, Dunn BE, et al. Seroprevalence of Helicobacter pylori infections in Thailand. J Infect Dis 1990;161:1237-41.

15. Chinprasatsak $S$, Wilairatana $P$, Visalwadi $P$, Sanguansri $P$, Batara L, Kityaporn D, et al. Helicobacter pylori prevalence in northeastern Thailand. Southeast Asian J Trop Med Public Health 1993;24:734-41.

16. Tan HJ, Goh KL. Changing epidemiology of Helicobacter pylori in Asia. J Dig Dis 2008;9:186-9.

17. Hooi JKY, Lai WY, Ng WK, Suen MMY, Underwood FE, Tanyingoh D, et al. Global prevalence of Helicobacter pylori infection: systematic review and meta-analysis. Gastroenterology 2017;153:420-9.

18. Patnayak R, Reddy V, Jena A, Rukmangadha N, Parthasarathy $\mathrm{S}$, Reddy MK. Utility of immunohistochemistry in demonstrating Helicobacter pylori. OGH Reports 2015;1:1-4. 
19. Yup LJ, Nayoung K. Diagnosis of Helicobacter pylori by invasive test: histology. Ann Transl Med 2015;3:1-8.

20. Lee HC, Huang TC, Lin CL, Chen KY, Wang CK, Wu DC. Performance of routine Helicobacter pylori invasive tests in patients with dyspepsia. Gastroenterol Res Pract 2013; 2013:184806

21. Iwahi $\mathrm{T}$, Satoh $\mathrm{H}$, Nakao M, Iwasaki T, Yamazaki T, Kubo K, et al. Lansoprazole, a novel benzimidazole proton pump inhibitor, and its related compounds have selective activity against Helicobacter pylori. Antimicrob Agents Chemother 1991;35:490-6.

22. Weil J, Bell GD, Powell K, Morden A, Harrison G, Gant PW, et al. Omeprazole and Helicobacter pylori: temporary suppression rather than true eradication. Aliment Pharmacol Ther 1991;5:309-13.

23. Andersen LP, Dorland A, Karacan H, Colding $H$, Nilsson $H O$, Wadström T, et al. Possible clinical importance of the trans- formation of Helicobacter pylori into coccoid forms. Scand J Gastroenterol 2000;35:897-903.

24. Nakao M, Tada M, Tsuchimori K, Uekata M. Antibacterial properties of lansoprazole alone and in combination with antimicrobial agents against Helicobacter pylori. Eur J Clin Microbiol Infect Dis 1995;14:391-9.

25. Nakao M, Malfertheiner P. Growth inhibitory and bactericidal activities of lansoprazole compared with those of omeprazole and pantoprazole against Helicobacter pylori. Helicobacter 1998;3:21-7.

26. Roberts SJ, Bateman DN. Prescribing of antacids and ulcerhealing drugs in primary care in the north of England. Aliment Pharmacol Ther 1995;9:137-43.

27. Malfertheiner P, Megraud F, O'Morain C, Bazzoli F, El-Omar E, Graham D, et al. Current concepts in the management of Helicobacter pylori infection: the Maastricht III Consensus Report. Gut 2007;56:772-81. 